\title{
Integrated central blood pressure-aortic stiffness risk score for cardiovascular risk stratification in chronic kidney disease
}

\author{
J Nemcsik ${ }^{1,2} *$ Á Tabák ${ }^{3,4 *}$, D Batta ${ }^{1 *}$, O Cseprekál $^{5}$, J Egresits $^{6}$, A Tislér $^{3}$ \\ ${ }^{1}$ Department of Family Medicine, Semmelweis University, Budapest, Hungary \\ ${ }^{2}$ Department of Family Medicine, Health Service of Zugló (ZESZ), Budapest, Hungary \\ ${ }^{3} 1$ st Department of Medicine, Semmelweis University, Budapest, Hungary \\ ${ }^{4}$ Department of Epidemiology and Public Health, University College London, London, UK \\ ${ }^{5}$ Department of Transplantation and Surgery, Semmelweis University, Budapest, Hungary \\ ${ }^{6}$ Department of Internal Medicine and Cardiology, Klinikum Klagenfurt, Klagenfurt am Wörthersee, Austria
}

Received: October 27, 2018

Accepted: November 6, 2018

Background and aims: The aim of this study was to develop an integrated central blood pressure-aortic stiffness (ICPS) risk score to predict cardiovascular events. Methods: It was a retrospective cohort study. A total of 100 chronic kidney disease (CKD) patients on conservative therapy were included. Pulse wave velocity (PWV), central systolic blood pressure (cSBP), and central pulse pressure (cPP) were measured. A score was assigned to tertiles of PWV (0-2), cPP (0-2), and cSBP ( 0 to the first and second and 1 to the third tertile) based on each parameter's ability to individually predict cardiovascular outcome. The sum of these scores and three ICPS risk categories as predictors were studied. Finally, we compared discrimination of the ICPS risk categories with PWV, cSBP, and cPP. Results: Adjusted for age and sex, patients in high and very high ICPS risk categories had increased cardiovascular risk (HR: 3.52, 95\% CI: 1.65-7.49; HR: 7.56, 95\% CI: 3.20-17.85, respectively). High and very high ICPS risk categories remained independent predictors in a model adjusted for multiple CV risk factors (HR: $4.58,95 \%$ CI: 1.65-7.49; HR: 8.56, 95\% CI: 3.09-23.76, respectively). ICPS risk categories (Harrell's C: $0.723,95 \%$ CI: 0.652-0.795) showed better discrimination than PWV (Harrell's C: $0.659,95 \%$ CI: $0.586-0.732, p=0.028$ ) and cSBP (Harrell's C: $0.660,95 \%$ CI: $0.584-0.735, p=0.008$ ) and there has been a tendency of significance in case of cPP (Harrell's C: $0.691,95 \%$ CI: $0.621-0.761, p=0.170$ ). Conclusion: The ICPS score may clinically importantly improve the identification of CKD patients with elevated cardiovascular risk.

Keywords: chronic kidney disease, central blood pressure, central pulse pressure, pulse wave velocity, cardiovascular outcome

\section{Introduction}

Given that cardiovascular (CV) diseases are still the leading causes of mortality worldwide and that an armamentarium of effective preventive medications is available, it is of utmost importance to accurately predict $\mathrm{CV}$ risk in different populations to increase the health benefits of $\mathrm{CV}$ prevention (5). Measurements of arterial stiffness and central hemodynamic status are contenders that may improve CV risk prediction over and above classical tools. These parameters have been extensively investigated in the past two decades. In all stages of

\footnotetext{
* These authors contributed equally to this work and are considered shared first authors.

Corresponding author: János Nemcsik, MD, PhD

Department of Family Medicine, Semmelweis University

4 Kútvölgyi Street, Budapest 1125, Hungary

Phone: +36 209827367; Fax: +36 13558530; E-mail: janos.nemcsik@gmail.com
} 
chronic kidney disease (CKD), arterial stiffness is an important risk factor for CV events and mortality (13).

The most important marker of arterial stiffness is the carotid-femoral pulse wave velocity (PWV). It was found to be predictive in different patient populations and was included in European hypertension guidelines since 2007. However, the most recent European guideline on CV risk prevention advised against its use for CV risk assessment in the general population (12).

Among parameters describing central hemodynamics, central systolic blood pressure (cSBP, a measure of pressure load) and central pulse pressure (cPP, describing pulsatility) seem to be the most promising, as they have better predictive values compared to brachial systolic and pulse pressure in some conditions $(1,7)$, although no additional advantage was found compared to brachial pressure in the Framingham Heart Study (8).

Another measure, the augmentation index (Aix), is a wave reflection parameter that also describes total peripheral resistance. It has also been reported to be an independent predictor of CV outcomes (6), but results are conflicting $(8,11)$.

Although most available literature on arterial stiffness investigates the predictive power of stiffness parameters individually, given that PWV, cSBP, cPP, and Aix can be obtained with most available devices at a single measurement, and that they reflect different aspects of the vasculature, it seems reasonable to combine their results into a single score to predict vascular events.

Our aims were to investigate the following in CKD patients on conservative therapy: (1) the predictive power of PWV, cSBP, cPP, and Aix individually for CV events; (2) to translate these parameters into simple scores based on their tertiles; (3) to establish and test for $\mathrm{CV}$ prediction an integrated parameter as the sum of these scores and based and these scores, different risk categories; and (4) to test whether the integrated score-based risk category concept improves CV prediction compared with its components separately.

\section{Methods}

It was a retrospective cohort study. Scientific results from this cohort were published previously $(1,10)$. Patients were recruited from two tertiary care nephrology outpatient clinics. Convenience sampling was used with the consecutive inclusion of CKD patients. None of the patients were hospitalized during baseline investigations. CKD patients at stages $1-5$, not on dialysis therapy, who gave written informed consent for participation, were included. Patients with atrial fibrillation or with frequent ventricular extrasystoles counteracting with pulse wave analysis were excluded. After baseline clinical, laboratory, arterial stiffness, and central hemodynamic measurements, patients were followed for a median of 67.6 months (interquartile range: 38.4-82.6). Follow-up data were collected between April 2007 and July 2014 by yearly telephone interviews either with the patients, their general practitioners, or treating physicians. All endpoint information was verified by original chart review. Follow-up was censored at the last occurrence of a documented CV event (acute coronary syndrome, heart failure requiring hospitalization, stroke or transient ischemic attack, or peripheral artery disease with the need for an intervention) or death due to the above CV causes.

The protocol was approved by the local ethical committees of the participating hospitals and was carried out in accordance with the tenets of the Declaration of Helsinki. All patients gave written informed consent before participation. 
Arterial stiffness, central hemodynamic, and blood pressure measurements

All measurements were performed between 10 a.m. and 12 p.m. Patients were allowed to take a non-standardized light breakfast and took their regular medications at least $3 \mathrm{~h}$ before the study measurements. They were asked to refrain from smoking on the day of the study and not to consume any caffeine-containing drinks at least $4 \mathrm{~h}$ before initiating the measurements. Arterial stiffness measurements and blood sampling were carried out on separate days within a week.

Arterial stiffness measurements were carried out in a temperature-controlled room $\left(24 \pm 1^{\circ} \mathrm{C}\right)$. Upon arrival after a 5 -min rest, two consecutive brachial blood pressure measurements were taken $1 \mathrm{~min}$ apart on each arm in the sitting position with a validated BpTru device (VSM Medtech, Vancouver, Canada). The mean value was calculated for each arm, and the higher of these was further taken as brachial systolic and diastolic blood pressure and heart rate. Subjects were then set in the supine position for a 10-min acclimatization period.

Arterial stiffness was measured with the "gold-standard" tonometric method, above the carotid and femoral sites, using the PulsePen device. We referred our previous publication for the PWV and cPP measurements (1). Aix was measured by automatic identification of the " 1 st shoulder" (inflexion point) on the averaged carotid pulse signal by the PulsePen software. The pressure amplitude following this point divided by the pulse pressure provided the Aix. CSBP was directly calculated from the carotid pulse waveform using the calibration considering brachial systolic and diastolic blood pressures.

\section{Epidemiologic and Laboratory data}

Baseline data on current smoking, any type of diabetes mellitus, hypertension, coronary artery disease (previous acute myocardial infarction or coronary intervention), chronic heart failure (previous diagnosis), peripheral arterial disease (documented by angiography or intervention), and cerebrovascular disease (previous stroke or transient ischemic attack) were collected by health record review.

Blood samples for the determination of blood cell count and hemoglobin, serum cholesterol, triglyceride, and low-density lipoprotein (LDL)-cholesterol were collected at baseline. Routine blood chemistry measurements were carried out directly after blood sampling on a Hitachi auto-analyzer. Baseline estimated glomerular filtration rate was calculated using the four-variable Chronic Kidney Disease Epidemiology Collaboration equation.

\section{Statistical analyses}

All data analyses were performed using SPSS 23 (SPSS Inc., Chicago, IL, USA) for Windows (descriptives and Cox regression analyses) or Stata version 13.1 (Harrell's C-statistics). Continuous data are given as mean and standard deviation (SD), or in case of evidence against a normal distribution, as a median and interquartile range.

The primary outcome of the study was the occurrence of the combined endpoint of CV events and CV mortality, as defined above.

To assess the predictive values of the studied parameters for the primary outcome, multiple failure times Cox proportional hazard regression analyses were used with conditional risk set modeling. This method accommodates for the fact that one patient may have had more than one event during follow-up. 
No a priori power calculations were carried out for the current analysis; however, the sample size for the original study was based on the observed differences and the distribution of one of the arterial stiffness measures (cPP) (1). A post hoc power calculation showed power values ranging from 0.60 to 0.97 for individual arterial stiffness parameters (as continuous variables) for the prediction of $\mathrm{CV}$ events.

Arterial stiffness and central hemodynamic parameters were analyzed both as continuous and categorical variables. For the former, these variables were transformed into $z$-scores to improve their comparability and thus the associations are given for one SD differences in PWV, cSBP, cPP, and Aix for the CV outcome. Model 1 was adjusted for age and sex, Model 2 was further adjusted for brachial systolic blood pressure, LDL-cholesterol, current smoking, diabetes, body mass index, known CV disease, and GFR-EPI. As in the cohort, all but one patient had hypertension, we omitted this variable from the adjustment.

Next, patients were divided into tertiles based on their PWV, cSBP, cPP, and Aix values, respectively. Survival was investigated using Kaplan-Meier analysis and Cox regressions similar to the ones described above, with arterial stiffness and central hemodynamic parameters as predictors and $\mathrm{CV}$ events or $\mathrm{CV}$ mortality as outcome. Polynomial and simple contrasts were performed to investigate the best scoring for these tertiles. According to these results, Aix was not related to $\mathrm{CV}$ outcome and was excluded from further analysis. There was a linear association between PWV and cPP and CV outcomes and accordingly 0,1 , and 2 points were given to the consecutive tertiles. As the risk of $\mathrm{CV}$ events or $\mathrm{CV}$ mortality only increased in the third tertile of $\mathrm{cSBP}, 0$ points were given to the first two tertiles and 1 point to the third.

The integrated central blood pressure-aortic stiffness (ICPS) score was calculated for each patient by summing the points based on tertiles (range: 0-5 points). Survival was investigated with Kaplan-Meier and Cox regression analyses (adjusted for age and sex) with ICPS score as the predictor and CV event or CV mortality as outcome. Given the limited statistical power of our relatively small sample size, patients were classified into three ICPS risk categories: average ( $0-2$ points), high (3-4 points), or very high (5 points). The predictive role of these risk categories were investigated in Kaplan-Meier curves and Cox regressions with adjustment (1) for age and sex and (2) with further adjustment for brachial systolic blood pressure, LDL-cholesterol, current smoking, diabetes, body mass index, CV disease, and GFR-EPI.

Finally, the ICPS risk categories and one SD change of each of its components (PWV, cSBP, and cPP) were analyzed in the same Cox regression model for CV outcomes. To investigate model discrimination, Harrell's concordance statistics were utilized.

As sensitivity analysis, all of the measurements were performed using Cox regression analyses, considering the occurrence of the first $\mathrm{CV}$ event instead of multiple failure time analysis as well.

\section{Results}

Of the 108 patients eligible for inclusion, five individuals declined participation. Furthermore, three patients were excluded because of missing baseline or follow-up data, leaving 100 subjects in the analytical sample.

Table I displays baseline characteristics, including concomitant diseases, traditional and non-traditional CV risk factors, and metabolic and vascular parameters. 
Table I. Baseline demographic, clinical, and laboratory characteristics $(n=100)$

\begin{tabular}{|c|c|}
\hline Male $(n)$ & 48 \\
\hline Age (years) & $66.00(58.25-75.00)$ \\
\hline BMI $\left(\mathrm{kg} / \mathrm{m}^{2}\right)$ & $27.63(25.24-30.49)$ \\
\hline Current smoker & 12 \\
\hline Diabetes mellitus & 44 \\
\hline Baseline cardiovascular disease & 64 \\
\hline Coronary artery disease & 13 \\
\hline Chronic heart failure & 19 \\
\hline Cerebrovascular disease & 24 \\
\hline Peripheral artery disease & 53 \\
\hline eGFR $\left(\mathrm{ml} / \mathrm{min}\right.$ per $\left.1.73 \mathrm{~m}^{2}\right)$ & $35.74(23.15-49.43)$ \\
\hline $\mathrm{Hgb}(\mathrm{g} / \mathrm{L})$ & $126.89(14.32)$ \\
\hline Chol $(\mathrm{mmol} / \mathrm{L})$ & $4.81(4.28-5.33)$ \\
\hline $\operatorname{Tg}(\mathrm{mmol} / \mathrm{L})$ & $1.80(1.15-2.60)$ \\
\hline $\mathrm{LDL}(\mathrm{mmol} / \mathrm{L})$ & $2.57(0.84)$ \\
\hline $\mathrm{SBP}(\mathrm{mmHg})$ & $135.50(120.31-145.44)$ \\
\hline DBP $(\mathrm{mmHg})$ & $73.12(9.70)$ \\
\hline $\mathrm{HR}(\mathrm{L} / \mathrm{min})$ & $62.25(57.50-72.63)$ \\
\hline PP (mmHg) & $60.38(50.56-70.38)$ \\
\hline $\mathrm{PWV}(\mathrm{m} / \mathrm{s})$ & $11.26(8.90-14.90)$ \\
\hline Aix $(\%)$ & $21.53(15.35-26.83)$ \\
\hline $\mathrm{cSBP}(\mathrm{mmHg})$ & $124.33(14.50)$ \\
\hline $\mathrm{cPP}(\mathrm{mmHg})$ & $48.58(42.75-60.38)$ \\
\hline
\end{tabular}

Categorical parameters are presented as $n$, numbers can be also considered as percentage. Continuous data are presented as mean (SD) or median (interquartile range). Aix: augmentation index; BMI: body mass index; Chol: cholesterol; cPP: central pulse pressure; cSBP: central systolic blood pressure; DBP: brachial diastolic blood pressure; eGFR: estimated glomerular filtration rate; Hgb: hemoglobin; HR: heart rate; LDL: low-density lipoprotein; $n$ : case number; PP: brachial pulse pressure; PWV: carotid-femoral pulse wave velocity; SBP: brachial systolic blood pressure; Tg: triglyceride

The causes of kidney disease were heterogeneous (number of cases in parentheses): glomerulonephritis $(n=14)$, diabetic nephropathy $(n=29)$, hypertensive nephrosclerosis $(n=17)$, chronic tubulointerstitial nephritis $(n=18)$, vascular cause $(n=6)$, polycystic kidney disease $(n=6)$, tumor $(n=1)$, and unknown $(n=9)$.

All but one patient received antihypertensive medication (case numbers in parentheses): angiotensin-converting enzyme inhibitors or angiotensin receptor blockers $(n=89)$, calcium channel blockers $(n=52)$, diuretics $(n=74), \beta$-receptor blockers $(n=54)$, $\alpha$-receptor 


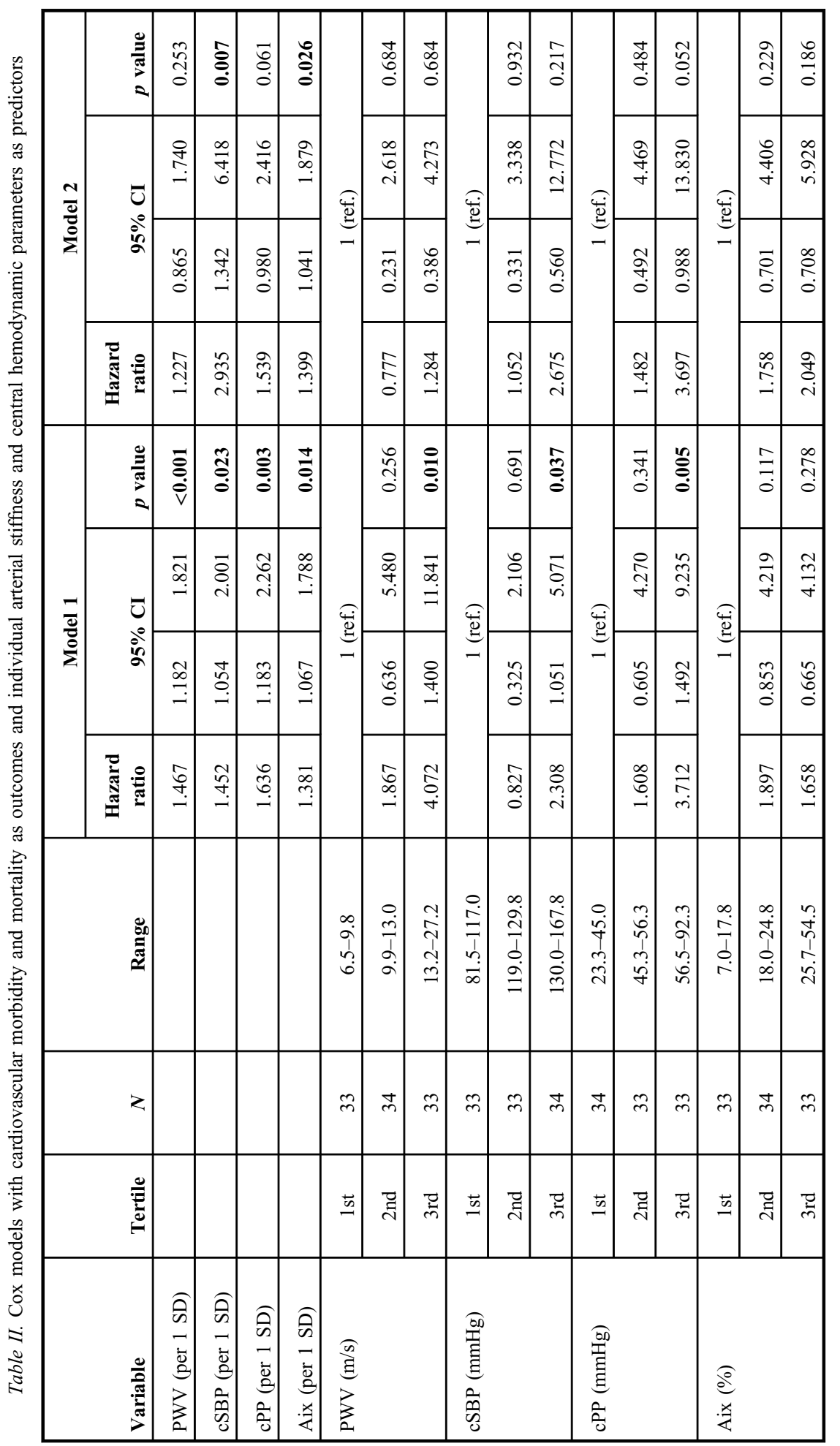

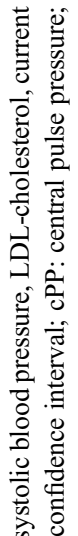
.ేّ 迅 范. 密苞 훙 总

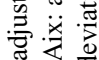
노물 吾恋 远唍

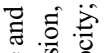
范 这芯 产芯芯 흘 줗 है चू

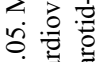
해

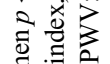
उ 莺客造 票告号 总

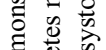
के क्ष อิ ขิ 㰴敦 

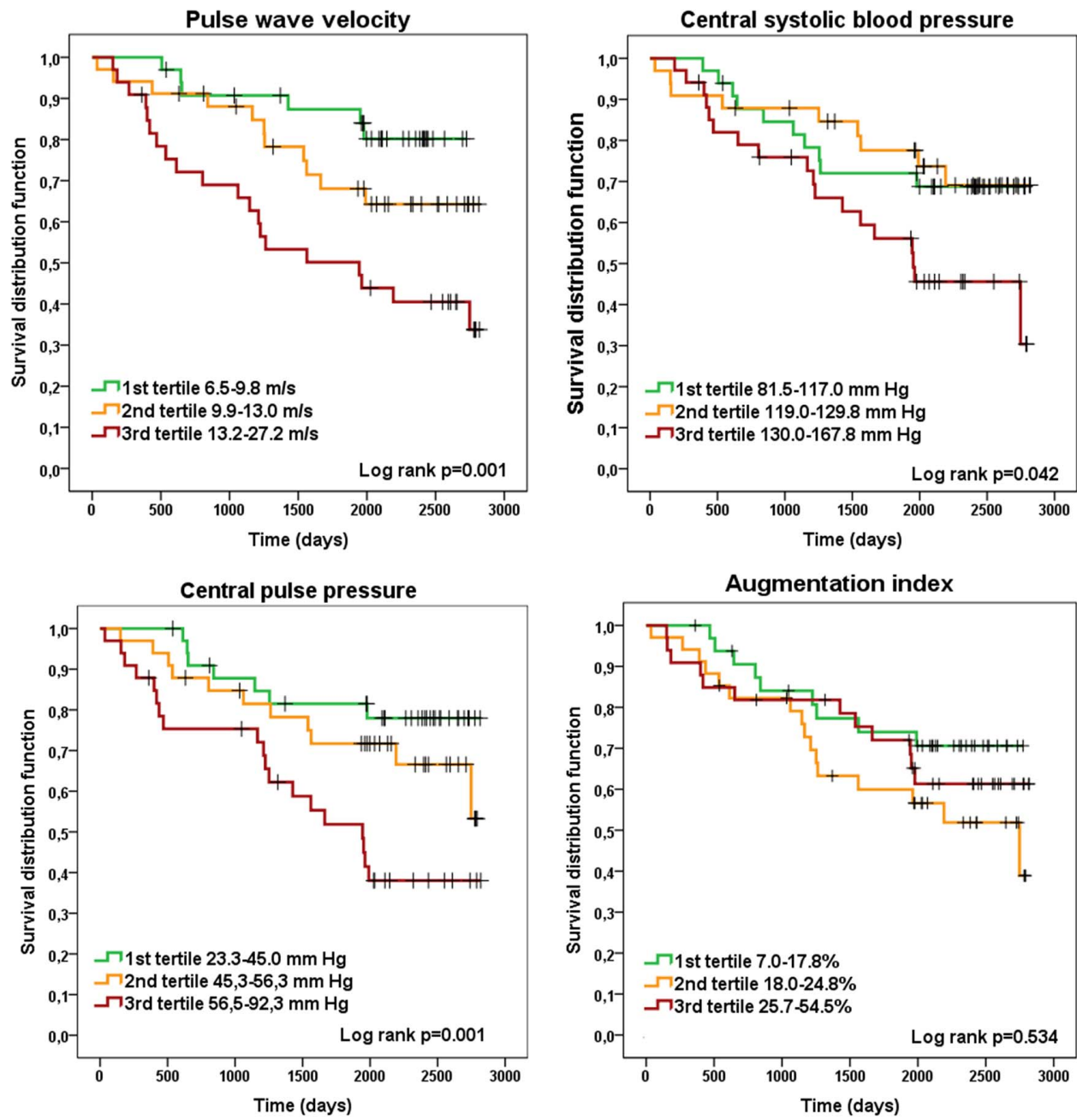

Fig. 1. Kaplan-Meier survival curves for each parameter studied with cardiovascular events (CV mortality and $\mathrm{CV}$ events) as outcomes. Panel A: pulse wave velocity; Panel B: central systolic blood pressure; Panel C: central pulse pressure; Panel D: augmentation index

blockers $(n=18)$, long-acting nitrate $(n=15)$, and centrally acting antihypertensive drugs $(n=13)$, either alone or in combination. Low dose aspirin was taken by $n=36$ patients, whereas $n=17$ individuals took clopidogrel. Sixty-one patients were on statin therapy.

On the whole, $n=37$ patients required erythropoietin-stimulating agents, $n=35$ received calcitriol, and $n=9$ needed calcium carbonate phosphate binder therapy.

During follow-up, $n=49 \mathrm{CV}$ events were recorded: $n=16$ patients died from CV causes (acute coronary syndrome $n=4$, stroke $n=3$, heart failure $n=8$, and peripheral artery disease $n=1$ ), and there were $n=33$ additional CV events (acute coronary syndrome $n=8$, stroke $n=6$, heart failure $n=12$, and peripheral artery disease $n=7$ ).

Table II demonstrates the association of PWV, cSBP, cPP, and Aix (per one SD change and per tertiles) with CV outcomes in models adjusted for age and sex or for traditional CV risk factors. All the four studied parameters were significantly related to $\mathrm{CV}$ outcomes in 
Model 1. In the further adjusted Model 2, the association of PWV and cPP was attenuated to non-significance, while cSBP and Aix showed significant associations. In the analyses of tertiles, PWV and cPP showed a linear association with the risk of CV outcomes, while for cSBP the association was non-linear: showing an increase only in the third tertile in Model 1 adjusted for age and sex. For Aix, no significant association was found, so this parameter was omitted from the ICPS score calculation. Further adjustment for traditional CV risk factors in Model 2 substantially attenuated the associations and none of them remained significant. Unadjusted associations are shown as Kaplan-Meyer curves for each tertile of all four parameters in Fig. 1.

Table III demonstrates hazard ratios for CV outcomes by ICPS risk scores and ICPS risk categories. The risk categories were derived from Cox models (Table III) and Kaplan-Meier (Fig. 2A) analyses by collapsing ICPS scores with similar hazard ratios and sufficient statistical

Table III. The relation of integrated central blood pressure-aortic stiffness (ICPS) risk score and ICPS risk categories with cardiovascular morbidity and mortality based on Cox proportional hazard regression models

\begin{tabular}{|c|c|c|c|c|c|}
\hline & $N$ & Hazard ratio & \multicolumn{2}{|c|}{$95 \%$ CI } & $p$ value \\
\hline \multicolumn{6}{|l|}{ ICPS risk score } \\
\hline \multicolumn{6}{|l|}{ Model 1} \\
\hline 0 & 18 & \multicolumn{4}{|c|}{1 (ref.) } \\
\hline 1 & 17 & 1.831 & 0.339 & 9.876 & 0.482 \\
\hline 2 & 16 & 1.528 & 0.233 & 10.018 & 0.659 \\
\hline 3 & 24 & 5.719 & 1.298 & 25.208 & 0.021 \\
\hline 4 & 13 & 4.236 & 0.849 & 21.131 & 0.078 \\
\hline 5 & 12 & 11.105 & 2.366 & 52.120 & 0.002 \\
\hline \multicolumn{6}{|c|}{ ICPS risk categories } \\
\hline \multicolumn{6}{|l|}{ Model 1} \\
\hline Average & 51 & \multicolumn{4}{|c|}{1 (ref.) } \\
\hline High & 37 & 3.517 & 1.650 & 7.494 & 0.001 \\
\hline Very high & 12 & 7.559 & 3.201 & 17.850 & $<0.001$ \\
\hline \multicolumn{6}{|l|}{ Model 2} \\
\hline Average & 51 & \multicolumn{4}{|c|}{1 (ref.) } \\
\hline High & 37 & 4.583 & 1.867 & 11.253 & 0.001 \\
\hline Very high & 12 & 8.563 & 3.086 & 23.758 & $<0.001$ \\
\hline Diabetes & 44 & 3.073 & 1.680 & 5.621 & $<0.001$ \\
\hline
\end{tabular}

Bold values demonstrate significance when $p<0.05$. Model 1 is adjusted for age and sex. Model 2 is adjusted for age, sex, brachial systolic blood pressure, LDL-cholesterol, current smoking, diabetes mellitus, body mass index, cardiovascular disease, and GFR-EPI. ICPS: integrated central blood pressure-aortic stiffness; CI: confidence interval 
A

Integrated central blood pressure aortic stiffness risk score

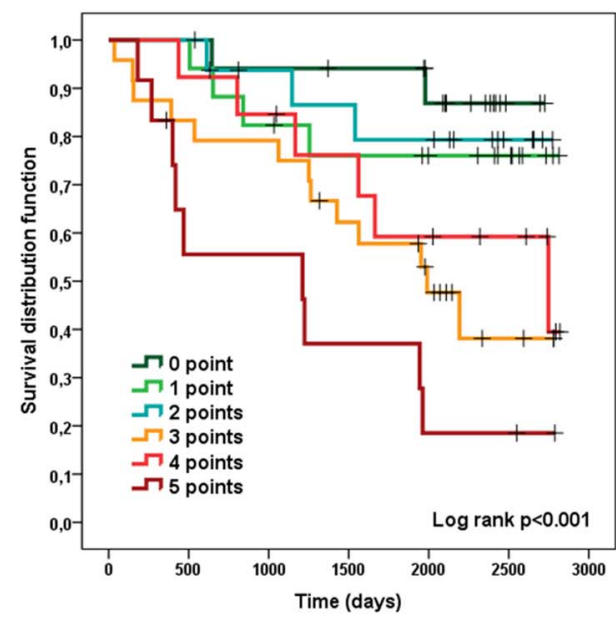

B Integrated central blood pressure aortic stiffness risk categories

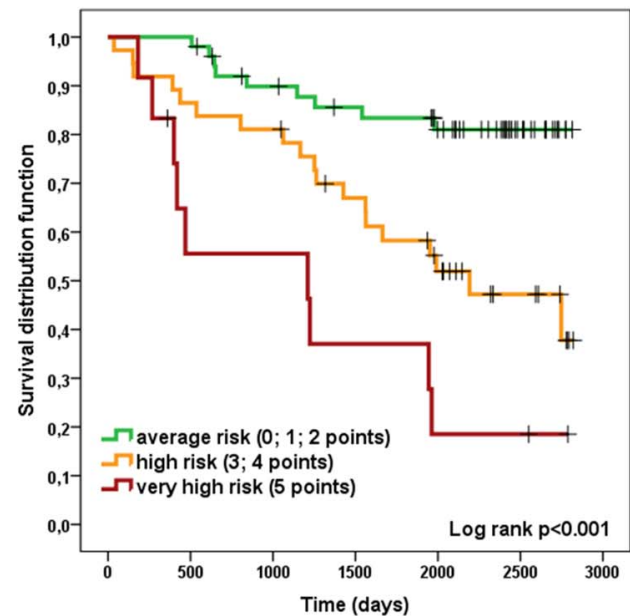

Fig. 2. Kaplan-Meier survival curves for the integrated central blood pressure-aortic stiffness (ICPS) risk scores and ICPS risk categories for cardiovascular events (CV mortality and CV events, adjusted for age and sex) as outcomes. Panel A: ICPS risk score groups; Panel B: ICPS risk categories

power. Almost half of the patients were classified into the high- and very high-risk categories. Kaplan-Meier survival curves for the three ICPS risk categories are shown in Fig. 2B.

Table III shows that the ICPS risk categories are strongly related to the CV outcomes even after adjustment for traditional CV risk factors. It is also notable that in Model 2, ICPS risk categories and diabetes were the only statistically significant predictors, with a higher risk in the high and very high ICPS risk categories compared to diabetes.

Table IV demonstrates the results of the comparison of the discriminative ability of the ICPS risk categories with the one SD change of PWV, cSBP, and cPP. All the parameters

Table IV. Comparison of the discriminative ability of the integrated central blood pressure-aortic stiffness risk categories with the one standard deviation change of pulse wave velocity, central systolic blood pressure, and central pulse pressure (Harrell's C-statistics)

\begin{tabular}{|l|c|c|c|c|c|}
\hline Variable & Coefficient & Standard error & \multicolumn{2}{|c|}{ 95\% CI } & $p$ value \\
\hline ICPS risk categories & 0.723 & 0.036 & 0.652 & 0.795 & $<\mathbf{0 . 0 0 1}$ \\
\hline PWV & 0.659 & 0.037 & 0.586 & 0.732 & $<\mathbf{0 . 0 0 1}$ \\
\hline cSBP & 0.660 & 0.038 & 0.584 & 0.735 & $<\mathbf{0 . 0 0 1}$ \\
\hline cPP & 0.691 & 0.035 & 0.621 & 0.761 & $<\mathbf{0 . 0 0 1}$ \\
\hline ICPS risk categories vs. PWV & 0.065 & 0.029 & 0.007 & 0.122 & $\mathbf{0 . 0 2 8}$ \\
\hline ICPS risk categories vs. cSBP & 0.064 & 0.024 & 0.017 & 0.110 & $\mathbf{0 . 0 0 8}$ \\
\hline ICPS risk categories vs. cPP & 0.032 & 0.023 & -0.014 & 0.079 & 0.170 \\
\hline
\end{tabular}

Bold values demonstrate significance when $p<0.05$. CI: confidence interval; ICPS risk categories: integrated central blood pressure-aortic stiffness risk categories; PWV: carotid-femoral pulse wave velocity; cSBP: central systolic blood pressure; cPP: central pulse pressure 
were adjusted for age and sex. ICPS risk categories were superior in the discrimination to PWV and cSBP and a tendency was also present in case of cPP, but the difference was not significant.

When, as sensitivity analysis, all the calculations were repeated with the closure of follow-up at the first event instead of multiple failure time analysis, similar results were found (data are available from authors by request).

\section{Discussion}

This study demonstrated that the concept of an integrated score based on arterial stiffness and central hemodynamic parameters (ICPS) is strongly related to incident CV events in CKD patients. According to our results, people in high and very high ICPS risk categories are at a remarkably high risk for $\mathrm{CV}$ events and have a risk that is stronger than that related to diabetes, the strongest single predictor among traditional risk factors in our cohort. In addition, it is better than PWV and cSBP and tends to be better than cPP, which suggests that it is worth adding together the predictive power of these parameters.

A recent consensus statement suggests that the combined assessment of more than one biomarker may improve CV outcome prediction (15). In line with this recommendation, this study investigated the combined effect of arterial stiffness and central hemodynamic parameters using a simple score that integrates the predictive information of individual biomarkers.

Available studies have conflicting results regarding the role of non-invasive markers of morphological or functional abnormalities of the arterial wall in relation to CV risk. In elderly patients of the Rotterdam study, the evaluation of carotid intima-media thickness (c-IMT), peripheral artery disease, or PWV marginally improved CV risk stratification over Framingham risk factors $(4,14)$. In contrast, in middle-aged subjects from the atherosclerosis risk in communities study, the detection of increased c-IMT and carotid artery plaques was associated with a significant $\sim 23 \%$ net reclassification index (9).

There are also some data available about the joint evaluation of different non-invasive hemodynamic biomarkers and their relation to CV outcomes. In the study of Wang et al. (16), cSBP was superior in CV outcome prediction compared to brachial systolic blood pressure or brachial or cPP. In the study of Holewijn et al. (3), using net reclassification improvement analysis, CV risk stratification improved by adding non-invasive vascular risk markers, such as PWV, Aix, or cSBP to traditional risk factors in women; however, the association was weaker in men and was limited to men at intermediate risk. These results suggest that the joint evaluation of different vascular biomarkers may have perspectives, but age and sex could influence the results. Although we adjusted for age and sex throughout this study, ICPS risk categories still remained robust predictors of $\mathrm{CV}$ events.

There are multiple potential advantages of the ICPS score concept. First, PWV, cSBP, and cPP can easily be estimated with most of the available devices (e.g., tonometric, mechanotransducer-based, or oscillometric) that measure arterial stiffness and use pulse wave analysis. The ICPS score is determined in a non-invasive manner without blood sampling, which is required for traditional risk scores. Furthermore, it could help to bridge the huge problem of diverging methodologies. Thanks to creative engineers, newer and newer devices are marketed that estimate these parameters in simpler ways, but the actual results of these devices are not interchangeable. Our ICPS score based on tertiles in a given population could be a universal parameter. Of course, the tertiles of each parameter should 
be defined for each device, but probably no equations are required to translate results between devices. Although our studied three parameters correlate with each other, but our results demonstrate, it is worth integrating them into one score as it can produce a very strong predictor parameter.

As the ICPS score is based on a limited sample of CKD patients, we do not recommend its calculation using the cutoff values from our sample, not even on CKD patients on conservative therapy. A valid risk score should be based on large databases with a much higher number of events that enable the investigation of each parameter involved in the score (2). However, as our ICPS risk categories in the present rudimentary form are much stronger predictors than diabetes in our cohort, our results in this form can generate important discussion and further studies. A great scientific potential of this concept is related to the fact that there are other cohorts in divergent races with available PWV, cSBP, and cPP measurements, so our finding on ICPS risk categories could easily be broaden for different patient populations. Such cohorts are, e.g., the Framingham Heart Study cohort (8) or the Nijmegen Biomedical Study (3).

There are some limitations of this study that has to be acknowledged. During tonometric arterial stiffness measurements, patients with atrial fibrillation are excluded because of methodological considerations, so a proportion of patients cannot be involved into our new risk stratification method. Due to the low number of participants and outcome events, this study is underpowered and thus the exact thresholds for scoring or the relative contribution of individual parameters could not have been exactly defined. Therefore, the aim of this study is not to define the final score but to report the possible advantages of this new concept of a combined risk score based on arterial stiffness and central hemodynamic parameters.

In conclusions, our integrated score and the constructed ICPS risk categories provided strong and robust association with $\mathrm{CV}$ outcomes in CKD patients on conservative therapy, which highlights the possible advantages of the combined measure of arterial stiffness and central hemodynamic parameters for $\mathrm{CV}$ risk prediction.

\section{Acknowledgements}

The authors would like to thank the contribution in patient recruitment to Dr. Lóránt Kerkovits, Dr. Adrienn Marton, and Dr. Zsófia K Németh. They would like to devote this manuscript to the memory of Prof. István Kiss, who contributed to this study, recently passed away. This study was supported by research grants from the Hungarian Kidney Foundation, Hungarian Society of Hypertension, and Hungarian Society of Nephrology. There has been no role of these grants in the design of the study and collection, analysis, and interpretation of data and in writing the manuscript.

\section{Conflict of interest}

The authors declare no conflict of interest.

\section{REFERENCES}

1. Cseprekal O, Egresits J, Tabak A, Nemcsik J, Jarai Z, Babos L, Fodor E, Farkas K, Godina G, Karpathi KI, Kerkovits L, Marton A, Nemcsik-Bencze Z, Nemeth Z, Sallai L, Kiss I, Tisler A: The significance of micro- and macrovascular biomarkers on cardiovascular outcome in chronic kidney disease: a prospective cohort study. J. Hum. Hypertens. 30, 449-455 (2016)

2. Fagard RH: Predicting risk of fatal cardiovascular disease and sudden death in hypertension. J. Hypertens. 35 , 2165-2167 (2017)

3. Holewijn S, den Heijer M, Kiemeney LA, Stalenhoef AF, de Graaf J: Combining risk markers improves cardiovascular risk prediction in women. Clin. Sci. (Lond). 126, 139-146 (2014) 
4. Kavousi M, Elias-Smale S, Rutten JH, Leening MJ, Vliegenthart R, Verwoert GC, Krestin GP, Oudkerk M, de Maat MP, Leebeek FW, Mattace-Raso FU, Lindemans J, Hofman A, Steyerberg EW, van der Lugt A, van den Meiracker AH, Witteman JC: Evaluation of newer risk markers for coronary heart disease risk classification: a cohort study. Ann. Intern. Med. 156, 438-444 (2012)

5. Lagerweij GR, de Wit GA, Moons KG, van der Schouw YT, Verschuren WM, Dorresteijn JA, Koffijberg H: A new selection method to increase the health benefits of CVD prevention strategies. Eur. J. Prev. Cardiol. 25, 642-650 (2018)

6. London GM, Blacher J, Pannier B, Guerin AP, Marchais SJ, Safar ME: Arterial wave reflections and survival in end-stage renal failure. Hypertension 38, 434-438 (2001)

7. McEniery CM, Cockcroft JR, Roman MJ, Franklin SS, Wilkinson IB: Central blood pressure: current evidence and clinical importance. Eur. Heart J. 35, 1719-1725 (2014)

8. Mitchell GF, Hwang SJ, Vasan RS, Larson MG, Pencina MJ, Hamburg NM, Vita JA, Levy D, Benjamin EJ: Arterial stiffness and cardiovascular events: the Framingham Heart Study. Circulation 121, 505-511 (2010)

9. Nambi V, Chambless L, Folsom AR, He M, Hu Y, Mosley T, Volcik K, Boerwinkle E, Ballantyne CM: Carotid intima-media thickness and presence or absence of plaque improves prediction of coronary heart disease risk: the ARIC (atherosclerosis risk in communities) study. J. Am. Coll. Cardiol. 55, 1600-1607 (2010)

10. Nemcsik J, Cseprekal O, Egresits J, Kielstein J, Kumpers P, Lukasz A, Tabak A, Marton A, Nemeth ZK, Jarai Z, Godina G, Sallai L, Farkas K, Kiss I, Tisler A: The role of laser doppler flowmetry tests, serum angiopoietin-2, asymmetric and symmetric dimethylarginine to predict outcome in chronic kidney disease. J. Hypertens. 35, 1109-1118 (2017)

11. Nemcsik J, Egresits J, El Hadj Othmane T, Fekete BC, Fodor E, Szabo T, Jarai Z, Jekkel C, Kiss I, Tisler A: Validation of arteriograph - a new oscillometric device to measure arterial stiffness in patients on maintenance hemodialysis. Kidney Blood. Press. Res. 32, 223-229 (2009)

12. Piepoli MF, Hoes AW, Agewall S, Albus C, Brotons C, Catapano AL, Cooney MT, Corra U, Cosyns B, Deaton C, Graham I, Hall MS, Hobbs FD, Lochen ML, Lollgen H, Marques-Vidal P, Perk J, Prescott E, Redon J, Richter DJ, Sattar N, Smulders Y, Tiberi M, van der Worp HB, van Dis I, Verschuren WM: 2016 European guidelines on cardiovascular disease prevention in clinical practice: the Sixth Joint Task Force of the European Society of Cardiology and Other Societies on Cardiovascular Disease Prevention in Clinical Practice (constituted by representatives of 10 societies and by invited experts) developed with the special contribution of the European Association for Cardiovascular Prevention \& Rehabilitation (EACPR). Eur. Heart J. 37, 2315-2381 (2016)

13. Taal MW: Arterial stiffness in chronic kidney disease: an update. Curr. Opin. Nephrol. Hypertens. 23, 169-173 (2014)

14. Verwoert GC, Elias-Smale SE, Rizopoulos D, Koller MT, Steyerberg EW, Hofman A, Kavousi M, Sijbrands EJ, Hoeks AP, Reneman RS, Mattace-Raso FU, Witteman JC: Does aortic stiffness improve the prediction of coronary heart disease in elderly? The Rotterdam Study. J. Hum. Hypertens. 26, 28-34 (2012)

15. Vlachopoulos C, Xaplanteris P, Aboyans V, Brodmann M, Cifkova R, Cosentino F, De Carlo M, Gallino A, Landmesser U, Laurent S, Lekakis J, Mikhailidis DP, Naka KK, Protogerou AD, Rizzoni D, Schmidt-Trucksass A, Van Bortel L, Weber T, Yamashina A, Zimlichman R, Boutouyrie P, Cockcroft J, O'Rourke M, Park JB, Schillaci G, Sillesen H, Townsend RR: The role of vascular biomarkers for primary and secondary prevention. A Position Paper from the European Society of Cardiology Working Group on Peripheral Circulation: Endorsed by the Association for Research into Arterial Structure and Physiology (ARTERY) Society. Atherosclerosis 241, 507-532 (2015)

16. Wang KL, Cheng HM, Chuang SY, Spurgeon HA, Ting CT, Lakatta EG, Yin FC, Chou P, Chen CH: Central or peripheral systolic or pulse pressure: which best relates to target organs and future mortality? J. Hypertens. 27, 461-467 (2009) 